\title{
SPECTRAL ANALYSIS OF (SEQUENCES OF) GRAPH MATRICES*
}

\author{
ANTONIO FRANGIONI ${ }^{\dagger}$ AND STEFANO SERRA CAPIZZANO
}

\begin{abstract}
We study the extreme singular values of incidence graph matrices, obtaining lower and upper estimates that are asymptotically tight. This analysis is then used for obtaining estimates on the spectral condition number of some weighted graph matrices. A short discussion on possible preconditioning strategies within interior-point methods for network flow problems is also included.
\end{abstract}

Key words. graph matrices, conditioning, preconditioning

AMS subject classifications. 05C50, 15A12, 65F 10

PII. S089547989935366X

1. Introduction. We study graph matrices coming from the application of interior-point methods [17, 14], which have grown a well-established reputation as efficient algorithms for large-scale problems. In these methods, at each step we have to solve linear systems of the form

$$
E \Theta E^{T} x=b,
$$

where $E$ is an $n \times m$ matrix and $\Theta$ is an $m \times m$ diagonal positive definite matrix. In most general-purpose solvers, these linear systems are solved by means of direct methods, typically the Cholesky decomposition preceded by a heuristic reordering of the columns of $E$ aimed at minimizing the "fill-in" [17]. We are interested in the possibility of using iterative methods instead. This can be beneficial in practice, especially in cases when $E$ is a sparse structured matrix [7] such as the node-arc incidence matrix of a graph $[15,16]$. However, these approaches can be competitive only if the rate of convergence of the iterative method is sufficiently high. This motivates our study of the extreme singular values of $E$ and of the spectral behavior of $E \Theta E^{T}$ since the convergence rate of iterative methods largely depends on the conditioning of the matrix. This analysis may have an interest for the development of preconditioners $[15,16]$ for the numerical solution to (1.1) through a preconditioned conjugate gradient (PCG) method (for the convergence theory of the PCG method, refer to $[3])$.

The paper is organized as follows. In section 2 we study the spectral properties (extremal behavior and conditioning) of $E E^{T}$ when $E$ is the node-arc incidence matrix of a directed graph. In section 3 we extend the analysis to "weighted" matrices of the form $E \Theta E^{T}$. Finally, in section 4 the connections between this analysis and some possible preconditioning strategies are briefly discussed.

2. Graph matrices. Let $H \equiv H_{n}=\left(\mathcal{U}_{n}, \mathcal{V}_{n}\right)$ be a directed graph with $n$ nodes $\mathcal{U}_{n}=\left\{u_{1}, \ldots, u_{n}\right\}$ and $m \operatorname{arcs} \mathcal{V}_{n}=\left\{v_{1}, \ldots, v_{m}\right\}$; its node-arc incidence matrix $E \equiv E_{n}=E\left(H_{n}\right)$ is the $n \times m$ matrix such that $E_{i j}=1$ if $v_{j}$ emanates from $u_{i}$, $E_{i j}=-1$ if $v_{j}$ terminates at $u_{i}$, and $E_{i j}=0$ otherwise.

${ }^{*}$ Received by the editors June 8, 1999; accepted for publication (in revised form) by L. El Ghaoui December 5, 2000; published electronically August 8, 2001.

http://www.siam.org/journals/simax/23-2/35366.html

†Dipartimento di Informatica, Corso Italia 40, 56100 Pisa, Italy (frangio@di.unipi.it).

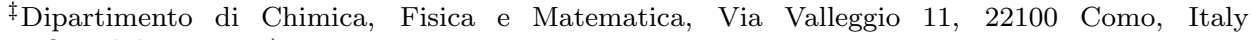
(serra@mail.dm.unipi.it). 
Here we analyze the spectral properties of sequences of matrices $\left\{E_{n} E_{n}^{T}\right\}_{n}$. Clearly, this both requires and implies the study of the spectra of (sequences of) graph matrices $\left\{E_{n}\right\}_{n}$. This analysis has an interest of its own, as demonstrated by the literature on the subject $[1,9]$. However, the usual approach has most often been of strongly combinatorial flavor and for a fixed graph size $n$. By contrast, our analysis focuses on asymptotical results, for which little or no previous work seems to have been published.

2.1. Preliminary results. The $E E^{T}$ matrix that we study is closely related to the Laplacian of an undirected graph $\bar{H} \equiv \bar{H}_{n}=\left(\overline{\mathcal{U}}_{n}, \overline{\mathcal{V}}_{n}\right)$ [1], i.e., the $n \times n$ matrix $L \equiv L_{n}=L\left(\bar{H}_{n}\right)$ such that $L_{i i}$ is the degree (number of incident arcs) of node $u_{i}$ and $L_{i j}$ for $i \neq j$ is -1 if the arc $(i, j)$ belongs to $\overline{\mathcal{V}}_{n}$ and zero otherwise. It is easy to prove the following relation between $L$ and $E E^{T}$.

Proposition 2.1. Given an undirected graph $\bar{H}=(\overline{\mathcal{U}}, \overline{\mathcal{V}})$, the directed graph $H=(\overline{\mathcal{U}}, \mathcal{V})$ with $\mathcal{V}=\{(i, j):(i, j) \in \overline{\mathcal{V}}, i<j\}$ has $E(H) E(H)^{T}=L(\bar{H})$.

In other words, the Laplacian of an undirected graph $\bar{H}$ can be obtained as $E(H) E(H)^{T}$, where $H$ is the directed graph obtained from $\bar{H}$ by orienting each arc in such a way that the head node is smaller than the tail node (with any fixed ordering of $\overline{\mathcal{U}}$ ). Conversely, the $E(H) E(H)^{T}$ matrix of a generic directed graph $H$ can be seen as being obtained from the Laplacians of two undirected graphs.

Proposition 2.2. Given a directed graph $H=(\mathcal{U}, \mathcal{V})$, the two undirected graphs $\bar{H}_{1}=\left(\mathcal{U}, \overline{\mathcal{V}}_{1}\right)$ and $\bar{H}_{2}=\left(\mathcal{U}, \overline{\mathcal{V}}_{2}\right)$ with

$$
\begin{aligned}
& \overline{\mathcal{V}}_{1}=\{(i, j):(i, j) \in \mathcal{V}, i<j\}, \\
& \overline{\mathcal{V}}_{2}=\{(i, j):(i, j) \in \mathcal{V}, j<i\}
\end{aligned}
$$

are such that $E(H) E(H)^{T}=L\left(\bar{H}_{1}\right)+L\left(\bar{H}_{2}\right)$.

Therefore, for the purpose of the analysis of the $E(H) E(H)^{T}$ matrices, a directed graph $H$ can be seen as the composition of two undirected graphs. One of the two graphs contains (as undirected edges) the arcs having a head node smaller than the tail node, while the other graph contains (as undirected edges) the arcs having a head node larger than the tail node.

Thus, Laplacians of undirected graphs and $E(H) E(H)^{T}$ matrices of directed graphs can be related through appropriate (de)orientation of the arcs. We will therefore be able to exploit some interesting results about the spectra of Laplacians such as the following.

TheOrem 2.3 (see [1]). For any undirected graph $\bar{H}, \lambda_{\max }(L(\bar{H})) \leq n$.

It is immediate to verify that summing all the rows of $E_{n}$ gives the null vector. This proves that $\lambda_{\min }\left(E_{n} E_{n}^{T}\right)=0$ and therefore $\sigma_{\min }\left(E_{n}\right)=0$ if $m \geq n$. However, if $H_{n}$ is a connected graph, then the matrix obtained by $E_{n}$ by eliminating any row has full rank. If $H_{n}$ has $k$ maximal connected components, then $E_{n}=E\left(H_{n}\right)$ is a block diagonal matrix with $k$ blocks; the minimal (maximal) singular value of $E_{n}$ is the minimum (maximum) among the minimal (maximal) singular values of the submatrices associated to the connected components. Hence, we can restrict our analysis to connected graphs. Note that $E_{n} E_{n}^{T}$ has exactly $k$ zero eigenvalues: by deleting $k$ appropriate rows of $E_{n}$ (one for each of the connected components), one can always obtain a matrix with no zero singular values.

We can always reorder the nodes and the arcs in such a way that the square submatrix $S \equiv S_{n}=S\left(H_{n}\right)$ made of the first $n-1$ rows of $E$ is nonsingular. In fact, 
$S_{n}$ is the node-arc incidence matrix of a spanning tree of $H_{n}$ less one row, for which the following results hold.

Proposition 2.4 (see [9]). $S_{n}$ is nonsingular and totally unimodular, i.e., the determinant of each square submatrix belongs to $\{ \pm 1,0\}$.

Proposition 2.5 (see [9]). The entries of $S_{n}^{-1}$ belong to $\{ \pm 1,0\}$.

2.2. Conditioning of trees. We start by studying the special case when $H$ is a tree, i.e., $m=n-1$ ( $H$ is connected). We do not require the arcs to have a specific orientation since the matrix $E^{\prime}=E\left(H^{\prime}\right)$, corresponding to the directed tree $H^{\prime}$ obtained from $H$ by reorienting the arcs, can be obtained from $E$ by right multiplication for an $m \times m$ diagonal $\{-1,+1\}$ matrix. By the singular value decomposition [4, 12], $E$ and $E^{\prime}$ have the same set of singular values; therefore, from the spectral viewpoint the directed tree $H^{\prime}$ can be considered a special representative of an equivalence class.

THEOREM 2.6. The conditioning of $S_{n}$ and $E_{n}$ satisfies

$$
\begin{gathered}
\bar{\delta}(n-1)^{1 / 2} \leq \kappa_{2}\left(S_{n}\right) \leq \sqrt{2 n}(n-1), \\
\frac{\bar{\delta}}{\sigma_{n-2}\left(S_{n}\right)} \leq \kappa_{2}\left(E_{n}\right) \leq \sqrt{2 n}(n-1),
\end{gathered}
$$

where $\bar{\delta}=\sqrt{\delta\left(H_{n}\right)}$ and $\delta\left(H_{n}\right)$ is the maximum degree among all nodes in $H_{n}$. Indeed

R1. $\sigma_{\min }\left(S_{n}\right) \leq(n-1)^{-1 / 2}$;

R2. $\sigma_{\min }\left(S_{n}\right) \geq(n-1)^{-1}$;

R3. $\sigma_{\max }\left(S_{n}\right) \leq(2 n)^{1 / 2}$;

R4. $\sigma_{\max }\left(S_{n}\right) \geq \bar{\delta} \geq \sqrt{2}$.

Proof.

Part R1. By the singular value decomposition of $S_{n}$

$$
\sigma_{\min }\left(S_{n}\right)=\inf _{\|x\|_{2}>0} \frac{\left\|x^{T} S_{n}\right\|_{2}}{\|x\|_{2}} \leq \frac{\left\|e^{T} S_{n}\right\|_{2}}{\|e\|_{2}}=\frac{1}{(n-1)^{1 / 2}},
$$

where $e$ is the vector of all ones.

Part R2. By Proposition 2.5, $\left|\left[S_{n}^{-1}\right]_{i, j}\right| \leq 1$, hence the entries of $B_{n}=S_{n}^{-T} S_{n}^{-1}$ cannot exceed $n-1$. Therefore, $\left\|B_{n}\right\|_{1} \leq(n-1)^{2}$; since $B_{n}$ is positive definite, its maximal eigenvalue coincides with its spectral norm and is less than its $\|\cdot\|_{1}$ norm, hence

$$
\lambda_{\max }\left(B_{n}\right) \leq(n-1)^{2},
$$

but $\lambda_{\max }\left(B_{n}\right)=\lambda_{\max }\left(S_{n}^{-1}\right)^{2}=\left(1 / \lambda_{\min }\left(S_{n}\right)\right)^{2}$.

Part R3. From Proposition 2.2, we know that there exist two undirected graphs $\bar{H}_{1}$ and $\bar{H}_{2}$ such that $E_{n} E_{n}^{T}=L\left(\bar{H}_{1}\right)+L\left(\bar{H}_{2}\right)$. Thus, using Theorem 2.3 and the fact that $S_{n}$ is a submatrix of $E_{n}$,

$$
\sigma_{\max }^{2}\left(S_{n}\right) \leq \sigma_{\max }^{2}\left(E_{n}\right)=\lambda_{\max }\left(E_{n} E_{n}^{T}\right) \leq \lambda_{\max }\left(L\left(\bar{H}_{1}\right)\right)+\lambda_{\max }\left(L\left(\bar{H}_{1}\right)\right) \leq 2 n .
$$

Part R4. Let $u_{h}$ be one of the nodes with maximum degree: it is always possible to reorient the arcs in such a way that $u_{h}$ becomes the root, i.e., it only has outgoing arcs. Then, let $e_{h}$ be the $h$ th vector of the canonical basis; by the singular value decomposition of $S_{n}$

$$
\sigma_{\max }\left(S_{n}\right)=\sup _{\|x\|_{2}>0} \frac{\left\|x^{T} S_{n}\right\|_{2}}{\|x\|_{2}} \geq \frac{\left\|e_{h}^{T} S_{n}\right\|_{2}}{\left\|e_{h}\right\|_{2}}=\frac{\sqrt{\delta\left(H_{n}\right)}}{1} .
$$

Note that $\delta\left(H_{n}\right) \geq 2$ since $H_{n}$ is connected. 
The bounds on the condition numbers of $S_{n}$ are simple consequences of R1$\mathbf{R} 4$ above. For the minimal and the maximal singular values of $E_{n}$, as well as its asymptotic conditioning, note that $S_{n}$ is a submatrix of $E_{n}$. We can apply a rewording of the Cauchy interlacing theorem that holds for non-Hermitian matrices [8]. In particular, the following relations hold:

$$
\begin{gathered}
\sigma_{n-2}\left(S_{n}\right) \geq \sigma_{\min }\left(E_{n}\right) \geq \sigma_{\min }\left(S_{n}\right), \\
\sigma_{\max }\left(S_{n}\right) \leq \sigma_{\max }\left(E_{n}\right) \leq \sqrt{2 n} .
\end{gathered}
$$

The estimates $\mathbf{R} \mathbf{1}-\mathbf{R} \mathbf{4}$ are, up to positive constants, tight: the following special structures are the "extremes" that prove it.

2.2.1. Linear trees. $H_{n}$ is a linear tree if it is a path, i.e., each node but two has exactly two incident arcs. We can assume that the path is oriented from the root to the unique leaf and that the nodes are ordered accordingly; thus, we obtain a bidiagonal matrix $E_{n}$. The corresponding $S_{n}$ is the $(n-1) \times(n-1)$ square Toeplitz matrix generated by the symbol $f(x)=1-e^{\mathbf{i} x}[18,6] . f(x)$ is weakly sectorial $[6]$ and has a zero of order 1 ; therefore, the analysis in [6] shows that

$$
\begin{gathered}
\sigma_{\min }\left(S_{n}\right) \sim n^{-1}, \\
\sigma_{\max }\left(S_{n}\right) \leq\|f\|_{\infty}=2, \\
\lim _{n \rightarrow \infty} \sigma_{\max }\left(S_{n}\right)=\|f\|_{\infty}=2 .
\end{gathered}
$$

Hence, $\mathbf{R} \mathbf{2}$ and $\mathbf{R} \mathbf{4}$ are tight (up to suitable multiplicative constants) for linear trees. These estimates can even be refined a little bit by studying the matrix $S_{n}^{T} S_{n}$. Direct calculation shows that

$$
S_{n}^{T} S_{n}=\left[\begin{array}{ccccc}
2 & -1 & 0 & \cdots & 0 \\
-1 & \ddots & \ddots & & 0 \\
0 & \ddots & & & \vdots \\
\vdots & & & 2 & -1 \\
0 & \cdots & 0 & -1 & 1
\end{array}\right]=T_{n-1}-e_{n-1} e_{n-1}^{T}
$$

where $T_{n-1}$ is the $(n-1) \times(n-1)$ Toeplitz matrix generated by the symbol $f(x)=$ $2-2 \cos (x) . T_{n-1}$ belongs to the $\tau$ algebra [5], so that its eigenvalues are explicitly known:

$$
\lambda_{\min }\left(T_{n-1}\right)=4 \sin ^{2}\left(\frac{\pi}{2 n}\right), \quad \lambda_{\max }\left(T_{n-1}\right)=4 \sin ^{2}\left(\frac{\pi(n-1)}{2 n}\right) .
$$

Note that $S_{n}^{T} S_{n} \leq T_{n-1}$ in the sense of the partial ordering of the Hermitian matrices; hence,

$$
\sigma_{\min }\left(S_{n}\right) \leq \pi / n, \quad \sigma_{\max }\left(S_{n}\right)=2-\epsilon_{n} \quad \text { with } \epsilon_{n} \geq \pi / n .
$$

Finally, since $E_{n}^{T} E_{n}=T_{n-1}$, we have

$$
\sigma_{\min }\left(E_{n}\right)=2 \sin \left(\frac{\pi}{2 n}\right), \quad \sigma_{\max }\left(E_{n}\right)=2 \sin \left(\frac{\pi(n-1)}{2 n}\right) .
$$

Remark 2.1. Observe that the constant $\|f\|_{\infty}=2$ is exactly the maximum node degree of a linear tree. Therefore, in the case of linear trees the lower bound in $\mathbf{R} 4$ is not tight and it can be replaced by $\delta\left(H_{n}\right)$ minus an asymptotically small quantity. 
2.2.2. Star trees. In the opposite direction, we have "concentrated" trees, the most concentrated one being the "star" tree where the root has $n-1$ sons. Let us choose any ordering for the nodes where the first node is the root, and let us order the arcs according to the chosen order of the nodes. The resulting $E_{n}$ is not lower triangular, but the corresponding $S_{n}$ has the following interesting structure:

$$
S_{n}=\left[\begin{array}{ccccc}
1 & 1 & 1 & \cdots & 1 \\
-1 & 0 & 0 & \cdots & 0 \\
0 & -1 & & & \vdots \\
\vdots & \ddots & \ddots & & \vdots \\
0 & \cdots & 0 & -1 & 0
\end{array}\right]
$$

This structure is close to that of the Frobenius matrices [4], and it is easy to prove that the characteristic polynomial is $p(\lambda)=\left(1+\lambda^{n}\right) /(1+\lambda)$. However, $S_{n}$ is "highly nonnormal" [4]; therefore the fact that all its eigenvalues have unitary modulus does not tell anything about its conditioning. As in the previous case, we can extract information on $S_{n}$ by studying the matrix

$$
S_{n} S_{n}^{T}=I_{n-1}+V_{n}, \quad \text { where } \quad V_{n}=\left[f \mid e_{1}\right] \cdot\left[e_{1} \mid g\right]^{T}
$$

and $g=-\sum_{j=2}^{n-1} e_{j}, f=(n-2) e_{1}+g$. Since $V_{n}$ has rank two, $S_{n} S_{n}^{T}$ has eigenvalues 1 with multiplicity $n-3$ plus two other values that can be explicitly calculated. The nonzero eigenvalues of $V_{n}$ are those of the $2 \times 2$ matrix [4]

$$
\left[e_{1} \mid g\right]^{T} \cdot\left[f \mid e_{1}\right]=\left[\begin{array}{cc}
n-2 & 1 \\
n-2 & 0
\end{array}\right] .
$$

Direct calculation yields

$$
\begin{gathered}
\lambda_{\max }\left(S_{n} S_{n}^{T}\right)=n-2+O\left(n^{-1}\right), \\
\lambda_{\min }\left(S_{n} S_{n}^{T}\right)=2 /(n-2)+O\left(n^{-2}\right) .
\end{gathered}
$$

Therefore

$$
\begin{aligned}
\sigma_{\max }\left(S_{n}\right) & =\sqrt{n-2+O\left(n^{-1}\right)}, \\
\sigma_{\min }\left(S_{n}\right) & =\sqrt{\frac{2}{n-2}+O\left(n^{-2}\right)},
\end{aligned}
$$

proving that $\mathbf{R} \mathbf{1}$ and $\mathbf{R} \mathbf{3}$ are tight up to suitable multiplicative constants.

Remark 2.2. The root of a star tree has degree $n-1$; hence, $\sigma_{\max }\left(S_{n}\right)=$ $\sqrt{n-2+O\left(n^{-1}\right)}=\sqrt{\delta\left(H_{n}\right)}+O(1)$ proves that $\mathbf{R} \mathbf{4}$ cannot be relaxed any further. Thus, $\bar{\delta}$ cannot be replaced by $\delta\left(H_{n}\right)$ as "substantially" done for linear trees (see Remark 2.1).

Remark 2.3. The case of "star" trees shows that the lower bound

$$
\frac{\bar{\delta}}{\sigma_{n-2}\left(S_{n}\right)} \leq \kappa_{2}\left(E_{n}\right)
$$


on the condition numbers in Theorem 2.6 is tight. Indeed $\bar{\delta}=\sqrt{n-1}, \sigma_{n-2}\left(S_{n}\right)=1$, and $E_{n} E_{n}^{T}=I_{n-1}+e e^{T}$ so that $\sigma_{\min }\left(E_{n}\right)=1, \sigma_{\max }\left(E_{n}\right)=\sqrt{n}$, and therefore $\kappa_{2}\left(E_{n}\right)=\sqrt{n}$ which is in good agreement with the bound.

Remark 2.4. Tightness of R1-R4 does not imply that the upper estimates on the spectral conditioning of $E_{n}$ in Theorem 2.6 are tight. In fact, "linear" trees have $O(n)$ condition numbers and "star" trees have $O(\sqrt{n})$ condition numbers, as opposed to the $O\left(n^{3 / 2}\right)$ bound given in the theorem. Finally, notice that the conditioning of $S_{n}$ and $E_{n}$ are asymptotically the same for "linear" trees while for "star" trees there is a substantially different behavior since $\kappa_{2}\left(E_{n}\right)=\sqrt{n}$ while $\kappa_{2}\left(S_{n}\right)$ grows as $n$.

2.3. Conditioning of graphs. The results of the previous section can be used in order to evaluate the extremal behavior of the singular values of $E\left(H_{n}\right)$ when $H_{n}$ is a generic graph with $n$ nodes. Since trees have been analyzed before, we will reduce the case of connected graphs to the case of trees.

Proposition 2.7. Let $H_{n}$ be a connected graph: then

$$
\sigma_{n-1}\left(E_{n}\right) \geq \max _{T \in T\left(H_{n}\right)} \sigma_{\min }\left(S_{n}(T)\right),
$$

where $T\left(H_{n}\right)$ is the set of the spanning trees of $H_{n}$.

Proof. Let $T$ be a generic spanning tree of $H_{n}$ : reorder the nodes and the arcs of $H_{n}$ in such a way that $T$ is represented by the first $n-1$ columns of $E_{n}$, and $S_{n}(T)$ is represented by the first $n-1$ rows and columns. Therefore, we have

$$
\begin{aligned}
\sigma_{n-1}\left(E_{n}\right) & =\sup _{\operatorname{dim} \mathrm{U}=n-1} \inf _{x \in U,\|x\|>0} \frac{\left\|x^{T} E_{n}\right\|_{2}}{\|x\|_{2}} \geq \inf _{y \in \mathcal{R}^{n-1},\|y\|>0} \frac{\left\|\left[y^{T}, 0\right] E_{n}\right\|_{2}}{\|[y, 0]\|_{2}} \\
& =\inf _{y \in \mathcal{R}^{n-1},\|y\|>0} \frac{\sqrt{\left\|y^{T} S_{n}(T)\right\|_{2}^{2}+\|w\|_{2}^{2}}}{\|y\|_{2}} \\
& \geq \inf _{y \in \mathcal{R}^{n-1},\|y\|>0} \frac{\left\|y^{T} S_{n}(T)\right\|_{2}}{\|y\|_{2}}=\sigma_{\min }\left(S_{n}(T)\right)
\end{aligned}
$$

From Proposition 2.7 and part R2 of Theorem 2.6, we obtain

$$
\sigma_{n-1}\left(E_{n}\right) \geq n^{-1} .
$$

On the other hand, using Proposition 2.2 and Theorem 2.3 as in part R3 of Theorem 2.6 , one obtains

$$
\sigma_{\max }\left(E_{n}\right) \leq(2 n)^{1 / 2} .
$$

As a consequence, the following theorem holds.

THEOREM 2.8. $\kappa_{2}\left(E_{n}\right)$ grows at most as $n^{3 / 2} \sqrt{2}$.

These bounds are asymptotically tight: linear trees realize (2.5), while (2.6) is realized by complete graphs. In fact, the matrix $E_{n} E_{n}^{T}$ corresponding to a complete graph $H_{n}$ is the circulant matrix $2\left(n I-e e^{T}\right)$, whose maximal eigenvalue is $2 n$ with multiplicity $n-1$ (all the nonzero vectors orthogonal to $e$ are eigenvectors associated to the eigenvalue $2 n$ ) and whose minimal eigenvalue is zero [10].

The bound on the condition number in Theorem 2.8 is asymptotically realized by a sequence of graphs $H_{n}$ with two components: a star tree $T_{n}^{1}$ with $\lfloor n / 2\rfloor$ nodes and a linear tree $T_{n}^{2}$ with $\lceil n / 2\rceil$ nodes. The maximal singular value of $E_{n}$ coincides with the one of $E\left(T_{n}^{1}\right)$, growing as $(n / 2)^{1 / 2}$, while the minimal nonzero singular value 
of $E_{n}$ coincides with the one of $E\left(T_{n}^{2}\right)$, collapsing to zero as $(n / 2)^{-1}$. Therefore, the spectral condition number behaves as $n^{3 / 2}$.

It is even possible to construct a sequence of trees having condition number asymptotic to $n^{3 / 2}$, answering in the positive to the question raised in Remark 2.4. Consider a sequence of trees $\hat{H}_{n+1}$ formed by the union of $T_{n}^{1}$ and $T_{n}^{2}$ with a new node $u$ and the two arcs that join $u$ with the roots of $T_{n}^{1}$ and $T_{n}^{2}$. We have

$$
\sigma_{\max }\left(\hat{E}_{n+1}\right) \geq \sigma_{\max }\left(E\left(T_{n}^{1}\right)\right)=\sqrt{\lfloor n / 2\rfloor-2+O\left(n^{-1}\right)} \sim n^{1 / 2} .
$$

Let the order of the nodes and the arcs of $\hat{H}_{n+1}$ be such that the first rows and columns are related to the linear tree $T_{n}^{2}$ :

$$
\hat{E}_{n+1}=\left[\begin{array}{cccccccc|cc}
1 & 0 & \cdots & 0 & & & & & 0 & -1 \\
-1 & 1 & \cdots & 0 & & & & & 0 & 0 \\
\vdots & \ddots & \ddots & \vdots & & & & & \vdots & \vdots \\
0 & \cdots & -1 & 1 & & & & & 0 & 0 \\
0 & \cdots & 0 & -1 & & & & & 0 & 0 \\
& & & & 1 & 1 & \cdots & 1 & -1 & 0 \\
& & & & -1 & 0 & \cdots & 0 & 0 & 0 \\
& & & & \vdots & \ddots & \ddots & \vdots & \vdots & \vdots \\
& & & & 0 & \cdots & -1 & 0 & 0 & 0 \\
& & & & 0 & \cdots & 0 & -1 & 0 & 0 \\
\hline 0 & \cdots & & 0 & 0 & \cdots & & 0 & 1 & 1
\end{array}\right]
$$

or, more compactly,

$$
\hat{E}_{n+1}=\left[\begin{array}{cc|cc}
E\left(T_{n}^{2}\right) & \mathrm{O} & 0 & -e_{1} \\
\mathrm{O} & E\left(T_{n}^{1}\right) & -e_{1} & 0 \\
\hline 0^{T} & 0^{T} & 1 & 1
\end{array}\right] .
$$

We have already seen in section 2.2.1 that $E\left(T_{n}^{2}\right)^{T} E\left(T_{n}^{2}\right)=T_{\bar{n}}$, where $\bar{n}=\lceil n / 2\rceil-1$ and $T_{h}$ is the $h \times h$ Toeplitz matrix generated by the symbol $f(x)=2-2 \cos (x)$, having

$$
\lambda_{\min }\left(T_{h}\right)=4 \sin ^{2}\left(\frac{\pi}{2(h+1)}\right) .
$$

Now, let $w \in \mathcal{R}^{\bar{n}}$ be the eigenvector of $T_{\bar{n}}$ corresponding to the minimum eigenvalue, and let $x \in \mathcal{R}^{n}$ be the vector $\left(1 /\|w\|_{2}\right)[w \mid 0]$ obtained by padding the normalized eigenvector with $\lfloor n / 2\rfloor+1$ zeroes. Since the $(n+1) \times n$ matrix $\hat{E}_{n+1}$ has full column rank, we have

$$
\begin{gathered}
\sigma_{\min }\left(\hat{E}_{n+1}\right)=\sigma_{n}\left(\hat{E}_{n+1}\right)=\inf _{\|y\|_{2}=1}\left\|\hat{E}_{n+1} y\right\|_{2} \\
\leq\left\|\hat{E}_{n+1} x\right\|_{2}=\frac{\left\|E\left(T_{n}^{2}\right) w\right\|_{2}}{\|w\|_{2}}=\frac{\sqrt{w^{T} E\left(T_{n}^{2}\right)^{T} E\left(T_{n}^{2}\right) w}}{\|w\|_{2}} \\
=\frac{\sqrt{w^{T} T_{\bar{n}} w}}{\|w\|_{2}}=2 \sin \left(\frac{\pi}{2\lceil n / 2\rceil}\right) \sim n^{-1},
\end{gathered}
$$

and therefore $\kappa_{2}\left(\hat{E}_{n+1}\right) \sim n^{3 / 2}$. 
3. Weighted graph matrices. We will now use the results of the previous section to study the spectral conditioning of (sequences of) weighted graph matrices $E_{n} \Theta E_{n}^{T}$. Let $\theta$ be the vector containing the diagonal elements of $\Theta$. By considering the Rayleigh quotient

$$
\frac{x^{T}\left(E_{n} \Theta E_{n}^{T}\right) x}{x^{T}\left(E_{n} E_{n}^{T}\right) x}=\frac{\sum_{i=1}^{m} y_{i}^{2} \theta_{i}}{\sum_{i=1}^{m} y_{i}^{2}}
$$

at any $x \notin \operatorname{Ker}\left(E_{n} E_{n}^{T}\right)$, it is easy to see that

$$
\begin{aligned}
& \lambda_{n-1}\left(E_{n} \Theta E_{n}^{T}\right) \geq \theta_{\min } \lambda_{n-1}\left(E_{n} E_{n}^{T}\right)=\theta_{\min } \sigma_{n-1}^{2}\left(E_{n}\right), \\
& \lambda_{\max }\left(E_{n} \Theta E_{n}^{T}\right) \leq \theta_{\max } \lambda_{\max }\left(E_{n} E_{n}^{T}\right)=\theta_{\max } \sigma_{\max }^{2}\left(E_{n}\right),
\end{aligned}
$$

where $\theta_{\min }$ and $\theta_{\max }$ are, respectively, the minimum and maximum elements of $\theta$ and where $\operatorname{Ker}(X)$ denotes the null space of a square matrix $X$. These estimates imply that the worst-case conditioning of $E_{n} \Theta E_{n}^{T}$ is in the order of $\left(\theta_{\max } / \theta_{\min }\right) n^{3}$.

Other estimates of the condition number of $E_{n} \Theta E_{n}^{T}$ can be obtained through the "decomposition to spanning trees" of $H_{n}$. For any subgraph $T$ of $H_{n}$, let us denote by $\mathcal{V}(T)$ the subset of $\mathcal{V}_{n}$ containing the $\operatorname{arcs}$ of $T$. Since the entries of $\theta$ (the diagonal elements of $\Theta$ ) are also indexed by arcs, we will denote by $\theta(T)[\Theta(T)]$ the subvector of $\theta$ (submatrix of $\Theta$ ) relative to the arcs in $\mathcal{V}(T)$ and by $\theta_{\max }(T)$ and $\theta_{\min }(T)$ its minimum and maximum elements, respectively. Thus, for any $T$

$$
E_{n} \Theta E_{n}^{T} \geq E(T) \Theta(T) E(T)^{T} \geq \theta_{\min }(T) E(T) E(T)^{T}
$$

in the sense of the partial ordering of the Hermitian matrices. Clearly, one is interested in "maximal" subgraphs $T$ of $H_{n}$, the obvious ones being spanning trees; therefore

$$
\lambda_{n-1}\left(E_{n} \Theta E_{n}^{T}\right) \geq \max _{T \in T\left(H_{n}\right)} \theta_{\min }(T) \lambda_{n-1}\left(E(T) E(T)^{T}\right),
$$

where $T\left(H_{n}\right)$ is the set of the spanning trees of $H_{n}$. The bound (3.3) can be strengthened by considering any set of disjoint spanning trees, i.e., the familiy

$$
D\left(H_{n}\right)=\left\{D \subseteq T\left(H_{n}\right): \mathcal{V}\left(T_{1}\right) \cap \mathcal{V}\left(T_{2}\right)=\emptyset \forall T_{1}, T_{2} \in D\right\} .
$$

For any $D \in D\left(H_{n}\right)$, one has

$$
E_{n} \Theta E_{n}^{T} \geq \sum_{T \in D} E(T) \Theta(T) E(T)^{T} \geq \sum_{T \in D} \theta_{\min }(T) E(T) E(T)^{T}
$$

and therefore

$$
\lambda_{n-1}\left(E_{n} \Theta E_{n}^{T}\right) \geq \max _{D \in D\left(H_{n}\right)} \sum_{T \in D} \theta_{\min }(T) \lambda_{n-1}\left(E(T) E(T)^{T}\right) .
$$

Note that the union of the subgraphs in $D$ need not cover all the arc set $\mathcal{V}_{n}$ of $H_{n}$. Actually, one may replace $T\left(H_{n}\right)$ in (3.4) with the set $A\left(H_{n}\right)$ of all acyclic subgraphs of $H_{n}$, allowing for more terms in the sum of (3.5). Unfortunately, all acyclic subgraphs $T$ which are not spanning trees have $\lambda_{n-1}\left(E(T) E(T)^{T}\right)=0$, so that all the corresponding terms give no contribution to the bound. 
Upper bounds on the maximum eigenvalue of $E_{n} \Theta E_{n}^{T}$ can be obtained with similar techniques. Consider the family of disjoint acyclic subgraphs covering all $\mathcal{V}_{n}$

$$
C\left(H_{n}\right)=\left\{C \subseteq A\left(H_{n}\right): \mathcal{V}\left(T_{1}\right) \cap \mathcal{V}\left(T_{2}\right)=\emptyset \forall T_{1}, T_{2} \in C, \bigcup_{T \in C} \mathcal{V}(T)=\mathcal{V}_{n}\right\} .
$$

Clearly, one has

$$
\lambda_{\max }\left(E_{n} \Theta E_{n}^{T}\right) \leq \min _{C \in C\left(H_{n}\right)} \sum_{T \in C} \theta_{\max }(T) \lambda_{\max }\left(E(T) E(T)^{T}\right) .
$$

The above estimates can be useful when designing preconditioners for the solution of (1.1) through a PCG method, as briefly discussed in the next section.

4. Conditioning and preconditioning. In this section we briefly discuss how the analysis of the previous paragraphs is related to the study of preconditioners for the solution of (1.1) through a PCG method. In the following, we will assume that one row (for each connected component of $H_{n}$ ) has been deleted from $E_{n}$, so that (1.1) is a full-rank system.

In the literature, tree-based preconditioners have been shown to be quite successful, in practice, for the solution of (1.1) within interior-point approaches to linear min-cost network flow problems $[15,16]$. These preconditioners are chosen as the matrices $S_{n} S_{n}^{T}$, where $S_{n}=S(T)$ corresponds to some spanning tree $T$ of $H_{n}$, usually an (approximate) maximum-weight spanning tree, the weight of arc $v_{i}$ being $\theta_{i}$. Besides working well in practice, this choice has a clear rationale from the analysis of interior-point methods in that, if the optimal solution of the underlying problem is unique, then the weights $\theta_{i}$ tend to zero on all arcs but those corresponding to the basic optimal solution [17] that form a spanning tree. However, another rationale for this choice is given by (3.3). In fact, it is well known that, in practice, having small eigenvalues is what hurts most the performance of a PCG method. Thus, spanning trees $T$ with large $\theta_{\min }(T)$ are presumably a good choice since

$$
\kappa_{2}\left(\left(E_{n}(T) E_{n}^{T}(T)\right)^{+} E_{n} \Theta_{n} E_{n}^{T}\right) \geq \theta_{\min }(T)
$$

with $X^{+}$denoting the pseudoinverse of Moore-Penrose of a matrix $X$ (see, e.g., [12]). Interestingly, the Kruskal algorithm that is typically used for computing the maximum-weight $T$ also gives the tree with largest $\theta_{\min }(T)$. This may be used to provide a more sophisticated convergence analysis for these methods.

Furthermore, (3.1) and (3.2) clearly imply that, using $E_{n} E_{n}^{T}$ as a preconditioner for (1.1), the spectral conditioning of the preconditioned matrix is limited by $\theta_{\max } / \theta_{\min }$. If the entries of $\theta$ would belong to a bounded interval $[r, R]$, with $r$ and $R$ positive constants independent on $n$, then $E_{n} E_{n}^{T}$ would be an optimal preconditioner for (1.1) [3], i.e., the number of PCG iterations required to achieve any chosen accuracy would be independent on $n$. Actually, it can be shown that the asymptotic behavior of the spectra of the preconditioner $E_{n} E_{n}^{T}$ describes the asymptotic behavior of the spectra of $E_{n} \Theta_{n} E_{n}^{T}$ for any "nondegenerating" sequence of positive $m(n)$ vectors $\theta_{n}$. Unfortunately, $\theta_{\max } / \theta_{\min }$ grows very fast during the iterations of the interior-point methods. However, blending a preconditioning technique using $E_{n} E_{n}^{T}$ and a (classical) $O(\sqrt{n})$ adaptive updating has recently led to an $O\left(\frac{n^{3}}{\log n} L\right)$ interiorpoint method for linear programming [2]. It is conceivable that similar techniques could be used to keep the quantity $\theta_{\max } / \theta_{\min }$ bounded. 
However, it is still not clear how to exploit the structure of $E_{n}$ in order to devise a fast algorithm for solving linear systems involving the matrix $E_{n} E_{n}^{T}$. In the specific case of local graphs [11], which generalize the idea of grid graphs, the self-similarity of the matrices $E_{n}$ and $E_{n^{\prime}}$, with $n^{\prime} \sim \theta n, \theta \in(0,1)$ independent of $n$, suggests the use of an algebraic multigrid method [13] since the matrix $E_{n^{\prime}}$ can be interpreted as a coarse grid version of the original matrix $E_{n}$.

Acknowledgment. We are grateful to Claudio Gentile for his contribution to a part of Theorem 2.6.

\section{REFERENCES}

[1] W.N. Anderson And T. Morley, Eigenvalues of the Laplacian of a graph, Linear and Multilinear Algebra, 18 (1985), pp. 141-145.

[2] K.M. Anstreicher, Linear programming in $O\left(\frac{n^{3}}{\log n} L\right)$ operations, SIAM J. Optim., 9 (1999), pp. 803-812.

[3] O. Axelsson and G. LindsköG, The rate of convergence of the preconditioned conjugate gradient method, Numer. Math., 52 (1986), pp. 499-523.

[4] R. Bhatia, Matrix Analysis, Springer-Verlag, New York, 1997.

[5] D. Bini and M. Capovani, Spectral and computational properties of band symmetric Toeplitz matrices, Linear Algebra Appl., 52/53 (1983), pp. 99-125.

[6] A. Böttcher and S. Grudsky, On the condition numbers of large semi-definite Toeplitz matrices, Linear Algebra Appl., 279 (1998), pp. 285-301.

[7] J. CAStro, A specialized interior-point algorithm for multicommodity network flows, SIAM J. Optim., 10 (2000), pp. 852-877.

[8] R. Courant And D. Hilbert, Methods of Mathematical Physics I, Interscience, New York, 1953.

[9] D. Cvetkovic, M. Doob, And H. Sachs, Spectra of Graphs, Academic Press, New York, 1979.

[10] P. Davis, Circulant Matrices, John Wiley and Sons, New York, 1979.

[11] A. Frangioni and S. Serra Capizzano, Matrix-Valued Linear Positive Operators and Applications to Graph Optimization, TR 04/99, 1999, Dip. di Informatica, Università di Pisa, Pisa, Italy.

[12] G.H. Golub And C.F. Van Loan, Matrix Computations, The Johns Hopkins University Press, Baltimore, MD, 1983.

[13] W. HackBusch, Multigrid Methods and Applications, Springer-Verlag, Berlin, 1985.

[14] Y. Nesterov and A. Nemirovskit, Interior-Point Polynomial Algorithms in Convex Programming, SIAM Stud. Appl. Math 13, SIAM, Philadelphia, 1994.

[15] M.G.C. Resende And G. Veiga, An implementation of the dual affine scaling algorithm for minimum-cost flow on bipartite uncapacitated networks, SIAM J. Optim., 3 (1993), pp. $516-537$.

[16] L.F. Portugal, M.G.C. Resende, G. Veiga, and J.J. Júdice, A truncated primal-infeasible dual-feasible network interior point method, Networks, 35 (2000), pp. 91-108.

[17] T. Roos, T. Terlaky, and J.-Ph. Vial, Theory and Algorithms for Linear Optimization: An Interior Point Approach, John Wiley and Sons, Chichester, UK, 1997.

[18] S. Serra, On the extreme eigenvalues of Hermitian (block) Toeplitz matrices, Linear Algebra Appl., 270 (1998), pp. 109-129. 